\title{
Economic Impact of a Specialist Outreach Team in Residential and Nursing Home Settings
}

\author{
Gerald Richardson ${ }^{1 *}$, Linda Davies², Belinda South², Rebekah Proctor ${ }^{3}$, Prof Alistair Burns ${ }^{4}$, Hilary \\ Stratton Powell ${ }^{4}$, Prof Nicholas Tarrier ${ }^{4}$ and Brian Faragher ${ }^{4}$ \\ ${ }^{1}$ Centre for Health Economics, University of York, Heslington, York YO10 4AW, UK ${ }^{2}$ Formerly Centre for Health Economics, University of York, \\ Heslington, York YO10 4AW ${ }^{3}$ Formerly Withington Hospital, Manchester, UK ${ }^{4}$ Withington Hospital, Manchester, UK
}

\begin{abstract}
Background: The results of a randomized controlled trial have indicated that a training and educational programme for staff in nursing or residential homes may result in reductions in levels of depression and levels of cognitive impairment for residents presenting with an active management problem. The training and educational intervention consisted of members of a hospital outreach team who presented a series of 1 hour seminars on topics which staff had indicated would improve their knowledge and skills.

Aims of the study: The aim of this study was to present an exploratory analysis of the impact on costs associated with providing an old age psychiatry outreach team giving training and education for staff in nursing and residential homes.

Method: For the economic study, a societal perspective was employed. Measures of resource use and costs to the health service, social and community services and the nursing and residential homes were analysed for 120 residents from 12 nursing or residential homes, as part of a randomized controlled trial to assess a training package provided in residential and nursing homes. Cost estimates were based on estimates from generalized estimated equations. To allow for clustering effects within homes, the unit of randomization was the home as opposed to the individual. To ensure models were correctly specified, several tests including the Ramsey RESET test were employed.

Results: There were no significant differences in the total cost per person in the homes that received the intervention and the control homes. This study has shown that the additional cost of providing the specialist outreach team was likely to be covered by reductions in the use of other resources such as GP visits to nursing and residential homes. Therefore, though the study had limitations, it appeared that improved care could be provided at little or no extra cost. Implications for Health Care Provision and Use: The evidence presented suggests that the specialist outreach team was unlikely to add to the total cost of caring for residents in nursing and residential homes. This finding combined with the benefits in terms of lower levels of depression and cognitive impairment suggested that the intervention was good value for money. The intervention should be considered for use in other nursing and residential homes. (C) 2000 John Wiley \& Sons, Ltd.
\end{abstract} * Correspondence to: Gerald Richardson MSc, Centre for Health Economics,
University of York, Heslington, York YO10 4AW, UK

Source of funding: This project was entirely funded by the NHS Executive under the primary/secondary care interface programme.
Received 18 May 2000; accepted 4 September 2000

\section{Introduction}

Residents who are elderly and mentally infirm and being cared for in nursing or residential homes often present staff with an 'active management problem': that is staff find them difficult to care for or cope with. Such residents may be confused or have a range of physical disabilities or could be suffering from depression, aggressive or challenging behaviour. Despite this, the level of training for staff in nursing and residential homes remains limited. ${ }^{1}$ The results of a clinical trial have shown that an old age psychiatry outreach team providing a training and educational programme for these staff results in improvements in terms of depression and cognitive impairment among residents. ${ }^{2}$ The cost of providing such a service could be cost additive but could also result in cost savings if the use of other services is reduced.

\section{Methods}

\section{Trial Design}

The full design of this study has been reported elsewhere. ${ }^{2}$ Briefly, the design of this study was a randomized controlled trial. Twelve nursing and residential homes in south Manchester were recruited into the study; each home provided ten residents for the study. The ten residents were selected by staff and were perceived as being difficult to manage and presenting an active management problem in the home. Six of the 12 homes received the intervention; the six that did not formed the control arm of the trial. Full ethical approval was obtained for the study, and consent was obtained from the residents and their relatives. The training and educational intervention consisted of members 
of a hospital outreach team who gave a series of seven 1 hour seminars on topics which staff had indicated would improve their knowledge and skills including management of dementia, aggression and screaming. Information on psychiatric disorders in old age and therapeutic approaches to care was also given as part of the seminar series. This was supported by the use of an experienced psychiatric nurse (HSP) who visited the intervention homes each week and gave advice to individual care workers. Each member of staff attended all of the seven seminars.

Data on resources used were collected retrospectively for each resident both before the intervention commenced and again after the entire intervention had been completed. In both cases resource use over the previous six-month period was collected.

\section{Measurement of Cost}

The cost analysis was of an exploratory nature and given the lack of published data should be used to inform future research. The costs of direct medical care were analysed for each resident. These costs included the costs of admissions to hospital, visits to and by the GP, visits by social and community health service staff (such as health visitors, social workers) as well as the cost of medication administered. The cost of each of these variables was estimated as the product of the unit cost and the level of resource use.

In addition, there was a cost associated with the intervention. Data on presenters and attendees of seminars were collected at the beginning of each seminar to estimate the additional cost of the intervention. The psychiatric nurse was employed for six months, half time at the midpoint of grade $\mathrm{G}$ on the nursing scales applicable from April 1996.

\section{Resource Use}

The homes involved in the study employed a Cardex system (or a variant on this system) to record drug use among residents. Resident records were maintained at each home; these recorded any hospital inpatient admission, outpatient appointment and/or consultation with any health professional. The health professionals included in the analysis were community psychiatric nurses (CPNs), district nurses (DNs), speech therapists, physiotherapists, general practitioners (both surgery visits and visits to the home), social workers, chiropodists and visits from a psychiatrist to the resident.

\section{Unit Costs}

Cost per day estimates for inpatient admissions was based on the local trusts financial returns; the same source was used for outpatient appointments. Unit costs for health and community based staff were estimated from the literature. Unit costs of medication were estimated from the British National Formulary. A full list of unit costs used and their sources is presented in Table $\mathbf{1}$.

For the purposes of this study, indirect costs due to lost productivity were not relevant as none of the residents were in employment. Costs that impacted on family members of residents, such as the cost of non-prescription medicine for the resident and the cost of travel to the residents' home, were not included in the analysis.

\section{Data Analytic Procedures}

The unit of randomization in this study was the nursing or residential home rather than the individual, necessitating the use of general estimated equation (GEE) models to allow for clustering effects within homes. Total mean costs were found to follow a positively skewed distribution and were therefore converted to natural logarithms for analysis and to aid with fitting an appropriate model; these results were then transformed back into the original units for presentation. Model specification was also assessed using Ramsey's RESET test. ${ }^{7}$ This is a general test designed to assess appropriateness of the chosen link function, inclusion of relevant regressors, omitted variable bias and normality of residuals.

A general to specific framework of entering all variables in the model and backwards eliminating variables for which their estimated coefficients were non-significant $(p>0.05)$ was adopted. Squared terms were included to allow for a more flexible functional form. At each stage the RESET test was applied until this indicated good specification. At this point, residual plots were used to confirm appropriateness of model chosen. All data were analysed using STATA version 5 (Stata Corporation, Texas).

\section{Results}

\section{Outcomes}

Clinical outcomes have been reported in more detail elsewhere. ${ }^{2}$ In summary, after adjusting for baseline values, residents in the intervention group showed a significantly lower deterioration in cognitive function than those in the control group $(p=0.002)$ over the study period as measured by a diagnostic algorithm AGECAT (Automatic geriatric examination for computer assisted taxonomy). ${ }^{8,9}$ No significant differences were found in activities of daily living, as measured by the Barthel Index, ${ }^{10,11}$ nor in behavioural characteristics of residents, as measured by the Crichton Royal Behavioural rating scale (CRBRS). ${ }^{12}$ One research psychologist (RP) assessed the residents independently of the training provider.

These results are similar to those obtained by Dean et al., ${ }^{13}$ who showed improvements in cognitive function after 3 months with a home based care programme for the elderly. Though the intervention group showed improved mental health functioning, no significant differences were found in the level of urinary incontinence between the groups.

All resource use data for the six-month period prior to the intervention were available. At the follow-up postintervention, there was incomplete information for 15 residents (nine in the control group and six in the intervention group, after adjustment for clustering effects the difference between groups was not significant). In 11 of these cases the person had died, two had been transferred to another 


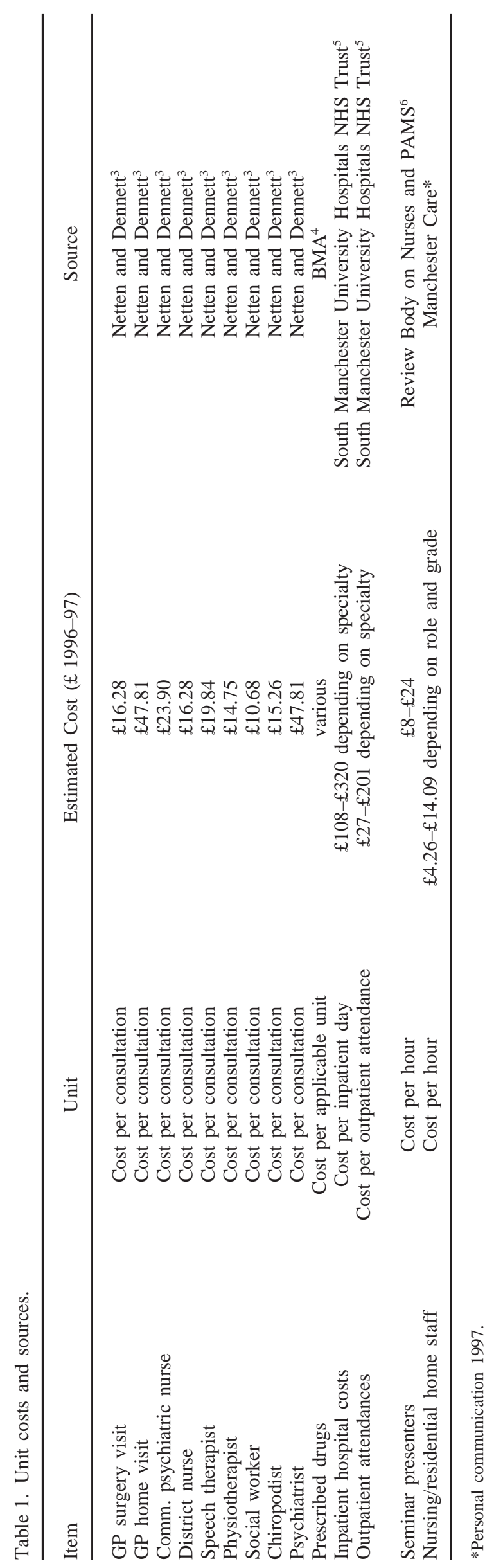


home or hospital, and two had withdrawn consent. There was no significant difference in mortality between the intervention and control groups. Residents who did not complete the study had a lower level of cognitive functioning than those who did. There were no differences in mortality or any of the clinical measures between residents who were in the intervention or control groups.

\section{Resource Use}

Table 2 shows the results for the economic variables for which modelling was appropriate. The other resource use variables measured did not exhibit sufficient variation in response, due to the large proportion of zero values, to allow more explicit modelling (for example, Poisson regression models failed to converge). For the district nurse variable, Poisson models were found to be unsatisfactory for inferential purposes due to non-normality of residuals, suggesting mis-specification bias.

The mean number of GP visits to resident homes was higher in the control group than in the intervention group both at baseline and at follow-up. Mean GP visits were not significantly different between study groups at baseline, but at the 6 month follow-up the mean count for the intervention group was significantly lower than that for the control group $(z=-2.06, p=0.04)$. After adjustment for baseline levels, the mean number of GP visits in the intervention homes was significantly less than for those in the control homes $(z=-2.21, p=0.027)$.

After adjustment for baseline levels, the mean number of chiropodist visits for the residents in the intervention homes was numerically less than for those in the control homes, but this difference was not statistically significant $(z=-0.72, p=0.475)$.

Table 3 shows the summary statistics for all the resource use variables.

It is worth noting that in the intervention group the number of GP visits to the home was reduced while GP surgery visits by residents increased. Similarly, outpatient visits increased in the intervention group while inpatient stays were reduced. This contrasts with the control group where GP home and surgery visits as well as inpatient and outpatient attendances increased. Though the number of non-zero observations for these data were small for most of these variables, and there were no significant differences in terms of ability to perform daily tasks as measured by the Barthel Index, these results suggested that the intervention was linked with a trend towards greater independence or mobility.

\section{Total Cost}

Total costs were found to have a non-normal distribution and so were transformed using natural logarithms for analysis. In addition, non-transformed costs were not amenable to modelling in that they failed the RESET test described above. The results, comparing intervention and control groups, are shown in Table 4.

A resident in the intervention group had a numerically slightly less mean cost at the six-month follow-up than one in the control group, controlling for all other factors mentioned in the above model. However this difference was not statistically significant $(z=-0.64, p=0.525)$. Female residents had a greater mean cost at follow-up than male residents, given that all other factors in the model are controlled for. This difference had a $p$-value of 0.058 and as such may warrant further investigation in future studies.

It should be noted that although this model passed the necessary assumptions for regression and the Ramsey RESET test $\left(z=-0.48, p=0.631\right.$ ), the low $R^{2}$ (approximately 0.07 ) associated with it implied that we should have little confidence in using this model for predictive purposes. Despite the fact that the model examined all possible sources of variance in the cost data available within the trial, it is clear that other factors explain the majority of the variation in cost data. Other variables reflecting, for example, socioeconomic background or support from family and friends may help explain more of this variation. However, a low $R^{2}$ with patient level cost data is not unusual in similar populations ${ }^{14,15}$ and does not invalidate the results. The fact that the model passed the RESET test means that the inference of a trend towards reduced costs was correct and that the model used was appropriate.

Performing univariate analysis on the raw data showed that the mean total costs were higher in the intervention group at baseline, but this difference was not statistically significant $(z=0.51, p=0.605)$. At six-month follow-up, the mean total costs were lower in the intervention group (including the cost of the intervention), but again this difference was not statistically significant $(z=-0.30$,

Table 2. Mean visits (95\% confidence limits) by treatment group for economic variables.

\begin{tabular}{|c|c|c|c|}
\hline \multirow[b]{2}{*}{ Variable } & \multicolumn{2}{|c|}{ Group } & \multirow{2}{*}{$\begin{array}{l}\text { Difference (control - intervention) (adjusted } \\
\text { - for baseline) }\end{array}$} \\
\hline & Intervention & Control & \\
\hline \multicolumn{4}{|c|}{ GP home visits } \\
\hline Baseline & $3.32(2.13$ to 5.18$)$ & 3.89 (2.98 to 5.07$)$ & - \\
\hline Follow-up & 2.81 (2.06 to 3.85$)$ & $4.28(3.27$ to 5.60$)$ & $0.70(0.51$ to 0.96$)$ \\
\hline \multicolumn{4}{|l|}{ Chiropodist } \\
\hline Baseline & 1.60 (1.23 to 2.06$)$ & 1.50 (1.04 to 2.14$)$ & - \\
\hline Follow-up & $1.23(0.87$ to 1.76$)$ & $1.50(0.95$ to 2.37$)$ & $0.82(0.49$ to 1.40$)$ \\
\hline
\end{tabular}


Table 3. Summary statistics for all economic variables for the control and treatment group. (Summary statistics for non-zero observations in brackets)

\begin{tabular}{|c|c|c|c|c|c|c|c|c|c|}
\hline \multirow[b]{2}{*}{ Service } & & \multicolumn{3}{|c|}{ Control } & \multirow{2}{*}{$\begin{array}{l}\text { No. of non- } \\
\text { zero } \\
\text { observations }\end{array}$} & \multicolumn{3}{|c|}{ Intervention } & \multirow{2}{*}{$\begin{array}{l}\text { No. of non- } \\
\text { zero } \\
\text { observations }\end{array}$} \\
\hline & & Minimum & Maximum & Mean & & Minimum & Maximum & Mean & \\
\hline \multirow[t]{2}{*}{ GP home visits } & Baseline & $0(1)$ & $10(10)$ & $3.86(4.38)$ & 45 & $0(1)$ & $13(13)$ & $3.30(3.79)$ & 47 \\
\hline & Follow-up & $0(1)$ & $12(12)$ & $4.24(4.41)$ & 49 & $0(1)$ & $15(15)$ & $2.81(3.45)$ & 44 \\
\hline \multirow[t]{2}{*}{ GP surgery visits } & Baseline & $0(2)$ & $2(2)$ & $0.04(2.00)$ & 1 & $0(1)$ & $4(4)$ & $0.19(1.67)$ & 6 \\
\hline & Follow-up & $0(1)$ & $2(2)$ & $0.10(1.67)$ & 3 & $0(1)$ & $9(9)$ & $0.28(3.00)$ & 5 \\
\hline \multirow[t]{2}{*}{ CPN } & Baseline & $0(1)$ & $6(6)$ & $0.22(3.67)$ & 3 & $0(1)$ & $16(16)$ & $0.48(6.5)$ & 4 \\
\hline & Follow-up & $0(6)$ & $7(7)$ & $0.25(6.50)$ & 2 & $0(7)$ & 18 (18) & $0.46(12.5)$ & 2 \\
\hline \multirow[t]{2}{*}{ District nurse } & Baseline & $0(1)$ & $13(13)$ & $1.35(3.14)$ & 22 & $0(1)$ & 12 (12) & $1.20(3.42)$ & 19 \\
\hline & Follow-up & $0(1)$ & $22(22)$ & $2.08(4.61)$ & 23 & $0(1)$ & $8(8)$ & $0.85(2.71)$ & 17 \\
\hline \multirow[t]{2}{*}{ Psychiatrist } & Baseline & $0(1)$ & $2(2)$ & 0.20 & 9 & $0(1)$ & $6(6)$ & $0.61(1.94)$ & 17 \\
\hline & Follow-up & $0(1)$ & $2(2)$ & $0.37(1.36)$ & 14 & $0(1)$ & $3(3)$ & $0.39(1.40)$ & 15 \\
\hline \multirow[t]{2}{*}{ Social worker } & Baseline & $0(1)$ & $2(2)$ & $0.06(1.5)$ & 2 & $0(1)$ & $1(1)$ & $0.09(1.00)$ & 5 \\
\hline & Follow-up & 0 & 0 & 0 & 0 & 0 & 0 & 0 & 0 \\
\hline \multirow[t]{2}{*}{ Speech therapist } & Baseline & $0(1)$ & $1(1)$ & $0.02(1.00)$ & 1 & 0 & 0 & 0 & 0 \\
\hline & Follow-up & $0(1)$ & $1(1)$ & $0.02(1.00)$ & 1 & 0 & 0 & 0 & 0 \\
\hline \multirow[t]{2}{*}{ Physiotherapist } & Baseline & $0(1)$ & $10(10)$ & $0.31(4.00)$ & 4 & $0(1)$ & $5(5)$ & $0.11(3.00)$ & 2 \\
\hline & Follow-up & $0(0)$ & $3(3)$ & $0.10(1.00)$ & 3 & $0(0)$ & $2(0)$ & $0.04(0.00)$ & 1 \\
\hline \multirow{2}{*}{ Outpatient visits } & Baseline & $0(1)$ & $2(2)$ & 0.20 & 9 & $0(1)$ & $2(2)$ & $0.30(1.07)$ & 15 \\
\hline & Follow-up & $0(1)$ & $4(4)$ & $0.41(1.50)$ & 14 & $0(1)$ & $4(4)$ & $0.35(1.46)$ & 13 \\
\hline \multirow[t]{2}{*}{ Inpatient visits } & Baseline & $0(1)$ & $4(4)$ & $0.18(3.00)$ & 3 & $0(3)$ & $36(36)$ & $2.09(16.14)$ & 7 \\
\hline & Follow-up & $0(10)$ & $91(91)$ & $3.75(38.20)$ & 5 & $0(2)$ & $15(15)$ & $0.43(7.67)$ & 3 \\
\hline
\end{tabular}

Table 4. Model obtained for natural log of total costs at follow-up adjusting for baseline and raw mean costs (95\% confidence limits) for both treatment groups at baseline and follow-up.

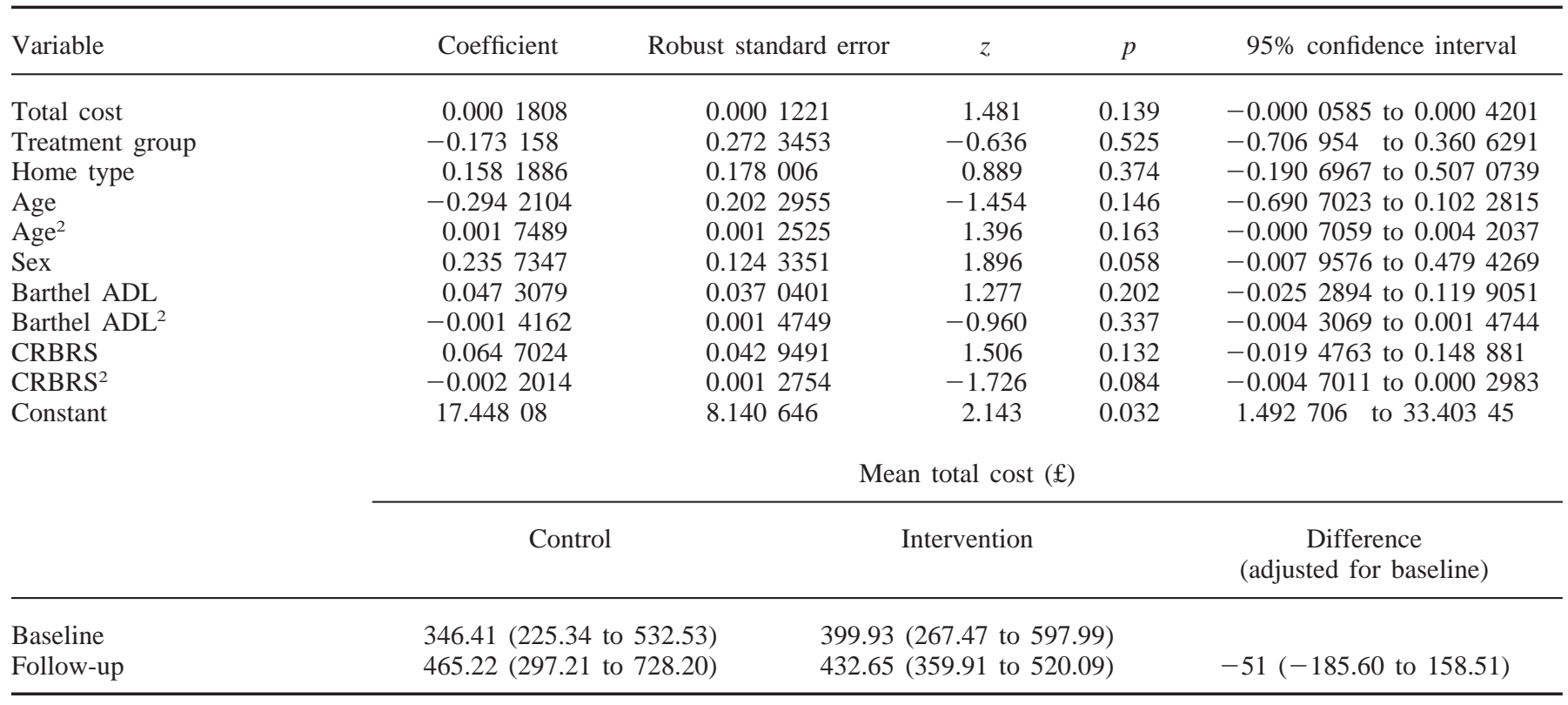

$p=0.76)$. The cost of the intervention was estimated as $£ 114$ per resident, which included the cost of staff attendance at the seminar series and the cost of employing the nurse. After adjustment for baseline levels, the mean total cost was numerically slightly less for residents in the intervention homes but was not statistically significant $(z=-0.57$, $p=0.572)$. The cost of medication showed a similar pattern to those of GP visits. Though differences were nonsignificant, the cost of medication fell in the intervention group from $£ 133$ to $£ 120$, while the same costs increased in the control group from $£ 163$ to $£ 171$.

These results agree with those obtained from the regression model for total cost. However, the results obtained from the regression model were more robust because parameters and standard errors were estimated accounting for other factors in the model such as age and sex. Intervention costs would have to increase dramatically for the intervention to be significantly cost additive. Given that these costs are 
based primarily on salaries of staff within the NHS and in nursing and residential homes, such increases are unlikely.

\section{Discussion}

Previous research has shown that a short seminar based intervention for staff in nursing and residential homes can alleviate correlates of cognitive impairment amongst residents. Staff in nursing and residential homes can easily be trained to develop skills that affect the quality of care in these settings.

This exploratory study has shown that the cost of this intervention was likely to be covered by reductions in the use of other resources such as GP visits to nursing and residential homes.

However, this study and the clinical study on which it was based had a relatively small sample size, based in one area of the UK and with a short follow-up period. It is conceivable that the benefits of this intervention may not be maintained in the long run. The effects of the intervention may diminish in time and 'refresher' seminars may be required. Thus the results may require confirmation in a larger trial with more homes in other geographical locations. In addition a trial with a longer period of follow-up of at least 12 months is required.

A further limitation of the study is that it considered only residents who presented an active management problem to members of staff. The effects on other residents were not monitored and so results may not be generalizable to other patient groups who do not present an active management problem. The impact of this programme on the amount of clinical time spent with residents and potentially therefore on the management of the homes was also not considered. It is feasible that improving the knowledge of care staff would reduce the need for input from clinical staff and release them to perform other tasks. However, it is also possible that, as knowledge increases, staff would identify more problems and request more clinical input.

It is also possible that, given that staff who received the intervention were not blinded, and that other staff in the homes were unlikely to be blind to the intervention, their expectations of the intervention may have biased the results. However, the expectations of staff may have led to more appropriate care for the residents. The results remain valid so long as these expectations and improved levels of care were independent of observation status. If observation of staff alters their expectations/behaviour, the bias remains and reduces the likelihood that improvements could be maintained over time.

\section{Implications for Health Care Provision and Use}

The intervention considered in this analysis is relatively inexpensive to provide and the additional cost is likely to be offset by reductions in the use of other resources such as visits to the home by a general practitioner.

The study used a societal perspective with the main costs being primary and secondary care. Decision makers in these environments should find these results of interest as should those organizing and providing care in nursing and residential homes.

This training and educational package has been shown to be effective in improving depression and cognitive behaviour in residents in nursing and residential homes. Despite its limitations, this study has demonstrated a trend towards reducing costs (including the cost of the intervention), and that it was unlikely to be cost additive. This provides limited evidence that such training programmes should be implemented in these settings, though the relatively small sample size means that further research is required to confirm these results.

\section{References}

1. Wilkin D, Evans G, Hughes B, Jolley D. The implications of managing confused and disabled people in non-specialist residential homes for the elderly. Health Trends 1982; 14: 98-100.

2. Proctor R, Stratton-Powell H, Burns A, Tarrier N, Richardson G, Davies L, South B, Farragher B. The impact of a specialist outreach team on the quality of nursing and residential home care: a randomised controlled trail. Lancet 1999; 354: 26-29.

3. Netten A, Dennet J. Unit Costs of Health and Social Care. University of Canterbury Personal Social Services Research Unit, 1996.

4. British Medical Association and the Royal Pharmaceutical Association of Great Britain. British National Formulary No. 33: 1997.

5. South Manchester University Hospitals NHS Trust. Provider Tariff 1996-97.

6. Review Body for Nursing Staff, Midwives, Health Visitors and Professions Allied to Medicine. Fourteenth Report Cm 3538: 1997.

7. Ramsey JB. Tests for misspecification errors in classical linear least squares regression analysis. J R Stat Soc B 1969; Vol 31: pp 350-371.

8. Copeland JRM, Kellaher M, Kellet M et al. A semi-structured clinical interview for the assessment of diagnosis and mental state in the elderly: the geriatric mental state schedule, I, development and reliability. Psychol Med 1976; 6: 439-449.

9. Copeland JRM, Dewey M, Griffiths-Jones H. Computerised psychiatric diagnostic system and case nomenclature for elderly subjects: GMS and AGECAT. Psychol Med 1986; 16: 89-99.

10. Mahoney FL, Barthel DW. Functional evaluation; the Barthel Index. Maryland State Med 1965; 14: 61-65.

11. Wade DT, Collin C. The Barthel ADL Index: a standard measure of physical disability? Int Disab Studies 1988; 10: 64-67.

12. Wilkin D, Thompson C. User's guide to dependency measures in elderly people. Social Services Monographs Sheffield. University of Sheffield, 1989.

13. Dean R, Briggs K, Lindesay J. The Domus philosophy: a prospective evaluation of two residential units for the elderly mentally ill. Int $J$ Geriatr Psychiatry 1993; 8: 807-817.

14. Chisholm D, Knapp MRJ, Astin J, Lelliott P, Audini B. The mental health residential care study: predicting costs from resident characteristics. Br J Psychiatry 1997; 170: 37-42.

15. Galster GC, Champney TF and Williams Y. Costs of caring for persons with long-term mental illness in alternative residential settings. Evaluation Program Planning 17: 239-248. 